\title{
Assessment of distribution of ventilation by electrical impedance tomography in standing horses
}

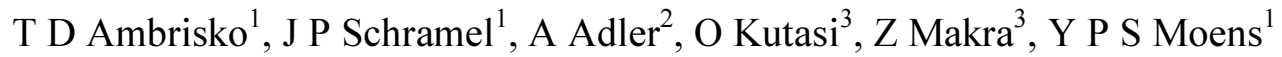

${ }^{1}$ Anaesthesiology and perioperative Intensive-Care Medicine, Department for Companion Animals and Horses, University of Veterinary Medicine, Vienna, Austria

${ }^{2}$ Department of Systems and Computer Engineering, Carleton University, Ottawa, Canada

${ }^{3}$ Equine Department and Clinic, Faculty of Veterinary Science, Szent István University, Üllö, Hungary

Current address of Dr. Kutasi: Large Animal Clinical Research Group, Hungarian Academy of Sciences-Szent István University, Üllö, Hungary

E-mail: tambrisko@hotmail.com

Short title: $\quad$ EIT in standing horses

Keywords: Distribution of ventilation, Electrical impedance tomography, Horse, Respiration 


\begin{abstract}
The aim was to evaluate the feasibility of using electrical impedance tomography (EIT) in horses. Thoracic EIT was used in nine horses. Thoracic and abdominal circumference changes were also measured with respiratory ultrasound plethysmography (RUP). Data were recorded during baseline, rebreathing of $\mathrm{CO}_{2}$ and sedation. Three breaths were selected for analysis from each recording. During baseline breathing, horses regularly took single large breaths (sighs), which were also analysed. Functional EIT images were created using standard deviations (SD) of pixel signals and correlation coefficients $(\mathrm{R})$ of each pixel signal with a reference respiratory signal. Left-to-right ratio, centre-of-ventilation and global-inhomogeneity-index were calculated. RMANOVA and Bonferroni tests were used $(P<0.05)$. Distribution of ventilation shifted towards right during sighs and towards dependent regions during sighs, rebreathing and sedation. Globalinhomogeneity-index did not change for SD but increased for R images during sedation. The sum of SDs for the respiratory EIT signals correlated well with thoracic $\left(r^{2}=0.78\right)$ and abdominal $\left(r^{2}\right.$ $=0.82)$ tidal circumferential changes. Inverse respiratory signals were identified on the images at sternal location and based on reviewing CT images, seemed to correspond to location of gas filled intestines. Application of EIT in standing non-sedated horses is feasible. EIT images may provide physiologically useful information even in situations, such as sighs, that cannot easily be tested by other methods.
\end{abstract}




\section{Introduction}

Horses have a unique respiratory system supporting the high athletic performance of these animals. Effective gas exchange has been demonstrated in horses while standing (Hedenstierna et al., 1987) and during high performance (Seaman et al., 1995) and also in conscious laterally recumbent ponies (Rugh et al., 1984). Nevertheless, horses when anaesthetised often develop pronounced maldistribution of ventilation and perfusion (V/Q) as a result of pulmonary atelectasis (Nyman and Hedenstierna, 1989). The ensuing venous admixture may cause a hypoxemic state in the perioperative period. This may play a role in the high rate of anaesthesia related mortality $(0.9 \%)$ (Johnston et al., 2002). Poor performance syndrome in horses is a major economical and welfare issue and respiratory diseases, such as recurrent airway obstruction (RAO) and inflammatory airway disease (IAD), are important causes of it (Van Erck-Westergren et al., 2013). Better understanding of the pathomechanism of V/Q disturbances in standing and anaesthetized horses may allow better prevention and treatment of these conditions; hence may decrease anaesthetic risk and improve welfare.

Information about the regional distributions of ventilation and perfusion are difficult to obtain and most of these techniques (e.g. using fluorescent microspheres, scintigraphy and computed tomography (CT)) have an invasive character and/or causing radiation exposure. electrical impedance tomography (EIT), a novel monitoring technology, may offer some advantages because it is a non-invasive, radiation-free, easily applicable and repeatable method for examining the aeration of the lungs (Adler et al., 2012; Costa et al., 2009b; Leonhardt and Lachmann, 2012). Its operation is based on measuring voltages between electrodes placed around the thorax while a small electrical current is introduced between two of these electrodes. A two 
dimensional impedance map can be reconstructed from these data representing regional changes in air volume at the EIT electrode plane. Creating such images at high repetition rate provides a video image. Despite, that the resolution of EIT is relatively low, regional distribution of ventilation obtained by EIT was favourably compared to those obtained by reference methods such as dynamic CT (Victorino et al., 2004), single-photon emission computed tomography (SPECT) (Hinz et al., 2003), positron emission tomography (PET) (Richard et al., 2009), hyperpolarized helium magnetic resonance imaging (MRI) (Dunster et al., 2012) and xenon CT (Elke et al., 2013) in different species.

To facilitate interpretation of EIT images of anaesthetized horses, such images need to be examined first for standing and conscious animals. However, the feasibility of using EIT in standing horses and the influence of clinical interventions (e.g. rebreathing and sedation) (Mazan and Hoffman, 2003) on EIT images are unknown.

The aim of this study was to examine the feasibility of using EIT and examine functional EIT images in standing horses during baseline, $\mathrm{CO}_{2}$-rebreathing and sedation.

\section{Methods}

\subsection{Animals}

The experimental protocol was approved by the institutional ethics committees and the national authorities in Austria (GZ 68205/171-II/106/2008) and in Hungary (22.1./1606/003/2009) because parts of the experiments were carried out in the veterinary universities of both countries. Nine warm-blood horses (four mares and five geldings) with a mean (SD) age of 11.6 (3.4) years 
and a bodyweight of $552(54) \mathrm{kg}$ were used. Calm animals had to be selected because the EIT device had relatively short cables and excessive movement of horses would have easily damaged the equipment. The physical examination, routine haematological and biochemical blood work and thoracic radiography were unremarkable. Seven horses had a history of RAO or IAD (Couetil et al., 2007) but they were currently free of respiratory symptoms at rest and lung function testing with the forced oscillation method (Young and Hall, 1989) did not reveal the presence of bronchoconstriction on the day before the experiment. Bronchoscopy and bronchoalveolar lavage (BAL) were performed one day after the experiment. Cytological examinations of the BAL fluid revealed that six horses had elevated (>10\%) (Corley and Stephen, 2008) neutrophil granulocyte count $(5,7,7,26,32,38,41,42$ and 43\%), indicating subclinical airway inflammation.

\subsection{Instrumentation}

Animals were fasted overnight before the experiment but water was not withheld. During the experiment the horses were restrained in a stock and 32 electrodes, attached to a custom-made rubber belt, were equidistantly placed around the thorax immediately behind the olecranon after clipping the area and using an electrode gel (Henry Schein). The electrodes were connected to an EIT device (model DX 1800, Timpel [former Dixtal]), which is described elsewhere (2009a; Costa et al., 2008). Respiratory ultrasound plethysmography (RUP) (Schramel et al., 2012b) was also used and served as an independent method for tidal volume measurement (Russold et al., 2013). The RUP belts were applied at the $11^{\text {th }}$ intercostal space and behind the last rib. The EIT and RUP data along with digital ECG signal were simultaneously downloaded and saved on a computer at $50 \mathrm{~Hz}$. 


\subsection{Experimental protocol}

Data were recorded in 5 min epochs. Initially, three epochs of baseline were consecutively recorded (baseline-1, baseline-2 and baseline-3). These were followed by rebreathing of $\mathrm{CO}_{2}$ via a tight-fitting face mask and a corrugated hose (rebreading-1) until the end-tidal $\mathrm{CO}_{2}$ pressure reached $60 \mathrm{mmHg}$ (iPM9800 patient monitor, Mindray). The animals were allowed to rest for 5 min (data not shown) then another epoch was recorded to confirm return to baseline (baseline-4). Then $0.5 \mathrm{mg} / \mathrm{kg}$ xylazine (Eurovet Animal Health) was injected into the jugular vein. Soon after two epochs were recorded consecutively (sedation-1 and sedation-2) and were followed by another rebreathing manoeuvre (rebreathing-2). Subsequently, all equipment was removed and the thoracic shapes at the site of the EIT electrode belt were modelled using gypsum cast.

\subsection{Creating an EIT mesh for horses}

In order to provide anatomically plausible EIT images there is a need for a pre-defined electronic image (mesh), which resembles the contour of the thorax and contains assumptions about internal organ location. The contour of a single representative horse was used to create such mesh for horses and organ location was assumed (Popesko, 1998). After the study, each individual gypsum contours were digitized, normalized and their mean was compared to the initial representative contour retrospectively.

\subsection{Post-hoc analysis of EIT data}

The thorax shape was projected into a rectangular image of $32 \times 32$ pixels (EIT Analysis Tools Beta 7.4.57, Timpel [former Dixtal]). In this image, 418 pixels were within the thorax boundary and contained impedance signals. 
Three breaths from each epoch were subjectively selected for analysis based on their similar shapes and amplitudes. Horses occasionally took single large breaths (sighs) during baseline. One sigh for each horse was also selected for analysis. Therefore, the following treatments were used for statistics: baseline-1, baseline-2, baseline-3, sigh, rebreathing-1, baseline-4, sedation-1, sedation-2 and rebreathing-2.

Three kinds of functional EIT images were created: full SD images (Fig. 2) containing the pixel-wise standard deviations of the impedance signals, R images (Fig. 3) containing Pearson correlation coefficients (linear regression analysis of each pixel and a reference respiratory signal located in the middle of a lung), and SD-Rpos images containing SDs for signals with positive R values (only those that positively correlate with ventilation).

The following variables were calculated from both the full SD and the SD-Rpos images: left to right (L/R) ratio, vertical geometric centre of ventilation (COV) (Frerichs et al., 2006), global inhomogeneity (GI) index (Zhao et al., 2009). The COV values expressed the distance from the sternum as $\%$ of the thoracic height. The GI indices were also calculated for the $\mathrm{R}$ images with positive or negative pixel values separately. In case of negative R values, the GI indices were multiplied by -1 .

The SD values of each image were summed up of for positively or negatively correlating signals separately. In case of the positively correlating signals, the sum of SDs represented tidal volume measurements (unitless) and for the negatively correlating signals it represented signal 
energy with so far unknown physiological meaning. Tidal differences in thoracic and abdominal RUP signals were also extracted.

After the calculations were complete, the SD and R images were normalized and averaged across horses and subsequently, on the basis of optical similarities they were further averaged among certain treatments (Fig. 2 and 3). The Matlab software (version 7.7.0.471 (R2008b), MathWorks) was used for calculations.

\subsection{Statistical analysis \\ Normality of the data was examined with the Shapiro-Wilk test. Missing data values were supplemented with an automatic multiple imputation method using five iterations. The data were compared across treatments using RM-ANOVA and Bonferroni test. Correlations between variables were examined using simple linear regression analysis. The test-wise $P$ value was less than 0.05 (SPSS, version 20, IBM).}

\section{Results}

The averaged thoracic contour was similar to the horse EIT mesh (Fig. 1). Data recording was omitted for two animals during rebreathing because of non-compliance. Sighs were present in the baseline recordings of all except two horses.

The averaged full SD and R images are shown on Fig. 2 and 3. In general, $78.5 \%$ (mean) of the pixels in each image correlated with the reference respiratory signal, $20 \%$ inversely correlated and $1.5 \%$ did not correlate. The numerical results are presented for the full SD and for 
the SD-Rpos images separately (Table 1). The distribution of ventilation shifted towards right (L/R ratio decreased) during sighs when compared to baseline on both kinds of SD images. The distribution of ventilation shifted towards ventral, dependent regions (COV decreased) on the full SD images during rebreathing-1. However, on the SD-Rpos images similar shift was detected during sigh, rebreathing-1 and sedation-1. The GI index for the SD images did not change during the study. Two way RM-ANOVA revealed that removal of the inverse respiratory signals decreased the $\mathrm{L} / \mathrm{R}$ ratio and GI indices but increased COV (since the inverse signals were located ventrally at the middle or slightly towards the left side).

Tidal volume (derived from EIT) increased during sighs, rebreathing and sedation-1 but the energy of inverse signals increased only during rebreathing (Fig. 4). These variables correlated $\left(r^{2}=0.35, P<0.001\right)$. The tidal volume measurements by EIT correlated with thoracic $\left(r^{2}=0.78, P<0.001\right)$ and abdominal $\left(r^{2}=0.82, P<0.001\right)$ tidal circumference changes measured by RUP.

Inhomogeneity (GI index) of the positive $\mathrm{R}$ values increased during sedation-1 compared to baseline indicating less good overall correlation with the reference respiratory signal (Fig. 5). This is visible on the $\mathrm{R}$ images as more bright-red or yellowish coloration during sedation when compared to the other treatments (Fig. 3). A similar, non-significant tendency can also be observed for the negative R values (Fig. 5 and Fig. 3).

Mean (SD) baseline respiratory rate was 14 (6) breaths/min which increased to 16 (2) breaths/min during rebreathing-1, decreased to 8 (2) breaths/min during sedation but was not 
different from baseline during rebreathing-2 [12 (2) breaths/min]. Thoracic radiographs that were made with an EIT electrode belt in situ indicated that the plane of the belt was near the interface of the cardiac apex and the diaphragm.

\section{Discussion}

This is the first study to report the distribution of ventilation in standing horses assessed by EIT. It appeared that the right lung received larger fraction of the tidal volume than the left one possibly because the heart is located more on the left side of the thorax; therefore the right lung is larger (Popesko, 1998). Slightly different L/R ratio values (mean values ranged 0.95-0.99) were published on standing Shetland ponies (Schramel et al., 2012a) but these differences may be explained by differences in thoracic conformations, physiological states (e.g. post-partum) and calculation methods.

Ventilation redistributed ventrally (dependent region) and towards right during sighs. Similarly, ventilation was ventrally redistributed during rebreathing-1 and sedation-1. These changes coincided with changes in tidal volumes but not consistently because during rebreathing2 the tidal volume increased largely but the distribution of ventilation did not change. The fact that the $2^{\text {nd }}$ rebreathing happened at a sedated state may have influenced this response. The GI index was designed to detect large changes in distribution of ventilation such as during one-lung ventilation or pulmonary diseases. Therefore, it is not surprising that this value did not change for the SD images in this study. Nevertheless, the GI values were similar to those reported in healthy anesthetized humans (Zhao et al., 2009). 
Ventilation and perfusion per unit of lung volume in standing horses was found to have a downward increasing gradient as measured by radioactive krypton scintigraphy (Amis et al., 1984). Such results cannot be compared to those of the present study because EIT cannot measure ventilation per unit of lung volume.

The existence of inverse signals has already been documented (Gomez-Laberge et al., 2011) and it was hypothesized to be caused by either the movement of abdominal gas (Hahn et al., 2008), by the filling of the heart during inspiration (Hahn et al., 2010; Pinsky, 2005) or by artefacts (Gomez-Laberge et al., 2011; Adler et al., 1996). However, venous filling of the heart is expected to have influence at the base of the heart and not near the apex as it was shown in this study. Similar, inverse signals were also observed dorsally but those signals had very low energy content and were anatomically located at the spinal process of the vertebra therefore these were assumed to be artefacts possibly caused by movement of the EIT electrodes during breathing (Adler et al., 1996; Gomez-Laberge et al., 2011) or by the reconstruction algorithm (Adler et al., 2009). Minor contribution of marginal artefacts to the ventral inverse signals cannot be excluded but it is hypothesized by the current authors that the tidal movement of abdominal gas might have been the major cause. In another study at our department (Reich et al., 2014) CT images of anesthetized ponies were examined. Apparently, large amount of intestinal gas was located a few $\mathrm{cm}$ caudal from the cardiac apex (Fig. 6) enclosed by the diaphragmatic flexure of the large colon. It is further hypothesized, that changes in energy content of these inverse signals may reflect changes in tidal diaphragmatic movement and the proximity of diaphragm to the EIT electrode plane. For example, increased energy of inverse signals as shown on Fig. 4 may suggest enhanced movement of the diaphragm during rebreathing. 
Functional EIT images, using the slopes of the regression lines, are widely used (Kuhnel et al., 1997; Pulletz et al., 2006; Pulletz et al., 2008; Hahn et al., 2008) but to authors' knowledge there is no publication using the Pearson $\mathrm{R}$ coefficients for this purpose. The $\mathrm{R}$ images may provide more information regarding the quality of correlation because the slopes are largely influenced by the amplitude of impedance changes. Changes in the homogeneity of $\mathrm{R}$ values during sedation are still awaiting explanation. This may indicate for example decreasing signalto-noise ratio. Whether such changes may be induced by unmatched changes in ventilation and perfusion (V/Q mismatch) needs to be further investigated. Sedation with an $\alpha_{2}$ agonist, such as xylazine, is known to cause V/Q mismatch, pulmonary hypertension, bronchodilation, increase in upper airway resistance and tidal volume and decrease in respiratory rate in horses (Nyman et al., 2009; Lavoie et al., 1992). These effects may have influenced our results.

A limitation of the study is that many horses had a chronic subclinical airway inflammation. Although, younger and healthier animals, which are less likely to suffer from inflammatory airway diseases, were available they could not be used because of their temperament. Because all of the horses underwent lung function testing before the experiment, the possible effect of their lung conditions on the distribution of ventilation is probably minimal. An EIT system with longer and detachable (more easily repairable) cables would facilitate the use of EIT in large animals.

Another limitation could be that EIT images represent a single cross section of the thorax and only a single EIT belt position was used in this study. However, the forelegs prevented more 
cranial placement of the electrode belt and in case of more caudal belt placement, abdominal organs would dominate at the EIT belt plane. Non-conventional placement of the electrode array (e.g. longitudinal or non-circular patterns) may also be considered but it demands custom image processing and such trials should be driven by goal oriented vision (e.g. whether the lungs, heart or the abdomen/diaphragm movement is to be tested).

\section{Conclusions}

Application of EIT in standing non-sedated horses is feasible and well tolerated provided they are used to stand in stocks. Baseline variables derived from functional EIT images were documented and changes in distribution of ventilation were detected in this study during sighs, rebreathing and sedation. Electrical impedance tomography is a promising tool to examine the distribution of ventilation the equine lungs because it is non-invasive, repeatable and able to capture instantaneous moments, such as sighs, which would not be possible with other methods. Nevertheless, subtracting inverse respiratory signals from the SD images may have to be considered because those may represent the movement of abdominal gas compartments and not lung ventilation. The hypothesis needs to be tested in further studies.

\section{Acknowledgements}

This study was supported by the University of Veterinary Medicine, Vienna, Austria (PP32011114). The authors wish to thank Dr. Erick Leon for technical support, Dr. Heidi Reich and colleagues (University of Veterinary Medicine, Vienna, Austria) for providing their CT images. Preliminary results were presented as an Abstract at the Conference of the Association of Veterinary Anaesthetists Spring Meeting, Nottingham, UK, $23^{\text {rd }}-26^{\text {th }}$ April 2014. None of the 
authors has any financial or personal relationships that could inappropriately influence or bias the content of the paper.

\section{References}

Adler A, Amato M B, Arnold J H, Bayford R, Bodenstein M, Bohm S H, Brown B H, Frerichs I, Stenqvist O, Weiler N and Wolf G K 2012 Whither lung EIT: where are we, where do we want to go and what do we need to get there? Physiological measurement 33 679-94

Adler A, Arnold J H, Bayford R, Borsic A, Brown B, Dixon P, Faes T J, Frerichs I, Gagnon H, Garber Y, Grychtol B, Hahn G, Lionheart W R, Malik A, Patterson R P, Stocks J, Tizzard A, Weiler N and Wolf G K 2009 GREIT: a unified approach to 2D linear EIT reconstruction of lung images Physiological measurement 30 S35-55

Adler A, Guardo R and Berthiaume Y 1996 Impedance imaging of lung ventilation: do we need to account for chest expansion? IEEE Transactions on Biomedical Engineering 43 414-20

Amis T C, Pascoe J R and Hornof W 1984 Topographic distribution of pulmonary ventilation and perfusion in the horse American journal of veterinary research 45 1597-601

Corley K and Stephen J 2008 Appendix In The Equine Hospital Manual Corley K and Stephen J eds Blackwell Publishing: Oxford, UK p 681

Costa E L, Borges J B, Melo A, Suarez-Sipmann F, Toufen C, Jr., Bohm S H and Amato M B 2009a Bedside estimation of recruitable alveolar collapse and hyperdistension by electrical impedance tomography Intensive care medicine 35 1132-7

Costa E L, Chaves C N, Gomes S, Beraldo M A, Volpe M S, Tucci M R, Schettino I A, Bohm S H, Carvalho C R, Tanaka H, Lima R G and Amato M B 2008 Real-time detection of pneumothorax using electrical impedance tomography Critical care medicine 36 1230-8

Costa E L, Lima R G and Amato M B 2009b Electrical impedance tomography Current opinion in critical care 15 18-24

Couetil L L, Hoffman A M, Hodgson J, Buechner-Maxwell V, Viel L, Wood J L and Lavoie J P 2007 Inflammatory airway disease of horses Journal of Veterinary Internal Medicine 21 356-61

Dunster K R, Friese M E, Fraser J F, Galloway G J, Cowin G J and Schibler A 2012 Ventilation distribution in rats: Part 2--A comparison of electrical impedance tomography and hyperpolarised helium magnetic resonance imaging Biomedical engineering online $\mathbf{1 1} 68$ 
Elke G, Fuld M K, Halaweish A F, Grychtol B, Weiler N, Hoffman E A and Frerichs I 2013 Quantification of ventilation distribution in regional lung injury by electrical impedance tomography and xenon computed tomography Physiological measurement 34 1303-18

Frerichs I, Dargaville P A, van Genderingen H, Morel D R and Rimensberger P C 2006 Lung volume recruitment after surfactant administration modifies spatial distribution of ventilation American journal of respiratory and critical care medicine 174 772-9

Gomez-Laberge C, Hogan M J, Elke G, Weiler N, Frerichs I and Adler A 2011 Data-driven classification of ventilated lung tissues using electrical impedance tomography Physiological measurement 32 903-15

Hahn G, Dittmar J, Just A and Hellige G 2008 Improvements in the image quality of ventilatory tomograms by electrical impedance tomography Physiological measurement 29 S51-61

Hahn G, Dittmar J, Just A, Quintel M and Hellige G 2010 Different approaches for quantifying ventilation distribution and lung tissue properties by functional EIT Physiological measurement 31 S73-84

Hedenstierna G, Nyman G, Kvart C and Funkquist B 1987 Ventilation-perfusion relationships in the standing horse: an inert gas elimination study Equine veterinary journal 19 514-9

Hinz J, Neumann P, Dudykevych T, Andersson L G, Wrigge H, Burchardi H and Hedenstierna G 2003 Regional ventilation by electrical impedance tomography: a comparison with ventilation scintigraphy in pigs Chest 124 314-22

Johnston G M, Eastment J K, Wood J L N and Taylor P M 2002 The confidential enquiry into perioperative equine fatalities (CEPEF): mortality results of Phases 1 and 2 Veterinary Anaesthesia and Analgesia 29 159-70

Kuhnel G, Hahn G, Frerichs I, Schroder T and Hellige G 1997 [New methods for improving the image quality of functional electric impedance tomography] Biomedizinische Technik $\mathbf{4 2}$ Suppl 470-1

Lavoie J P, Pascoe J R and Kurpershoek C J 1992 Effect of head and neck position on respiratory mechanics in horses sedated with xylazine American journal of veterinary research $\mathbf{5 3}$ $1652-7$

Leonhardt S and Lachmann B 2012 Electrical impedance tomography: the holy grail of ventilation and perfusion monitoring? Intensive care medicine 38 1917-29

Mazan M R and Hoffman A M 2003 Clinical techniques for diagnosis of inflammatory airway disease in the horse Clinical Techniques in Equine Practice 2 238-57

Nyman G and Hedenstierna G 1989 Ventilation-perfusion relationships in the anaesthetised horse Equine veterinary journal 21 274-81 
Nyman G, Marntell S, Edner A, Funkquist P, Morgan K and Hedenstierna G 2009 Effect of sedation with detomidine and butorphanol on pulmonary gas exchange in the horse Acta veterinaria Scandinavica $\mathbf{5 1} 22$

Pinsky M R 2005 Cardiovascular issues in respiratory care Chest 128 592S-7S

Popesko P 1998 Teil 2. Brust und Bauchhöhle In Atlas der topographischen Anatomie der Haustiere Popesko P ed Enke: Stuttgart p 134

Pulletz S, Elke G, Zick G, Schadler D, Scholz J, Weiler N and Frerichs I 2008 Performance of electrical impedance tomography in detecting regional tidal volumes during one-lung ventilation Acta anaesthesiologica Scandinavica 52 1131-9

Pulletz S, van Genderingen H R, Schmitz G, Zick G, Schadler D, Scholz J, Weiler N and Frerichs I 2006 Comparison of different methods to define regions of interest for evaluation of regional lung ventilation by EIT Physiological measurement 27 S115-27

Reich H, Moens Y, Braun C, Kneissl S, Noreikat K and Reske A 2014 Validation study of an interpolation method for calculating whole lung volumes and masses from reduced numbers of CT-images in ponies The Veterinary Journal (in press)

Richard J C, Pouzot C, Gros A, Tourevieille C, Lebars D, Lavenne F, Frerichs I and Guerin C 2009 Electrical impedance tomography compared to positron emission tomography for the measurement of regional lung ventilation: an experimental study Critical care 13 R82

Rugh K S, Garner H E, Hatfield D G and Herrold D 1984 Arterial oxygen and carbon dioxide tensions in conscious laterally recumbent ponies Equine veterinary journal 16 185-8

Russold E, Ambrisko T D, Schramel J P, Auer U, Van Den Hoven R and Moens Y P 2013 Measurement of tidal volume using respiratory ultrasonic plethysmography in anaesthetized, mechanically ventilated horses Veterinary Anaesthesia and Analgesia 40 $48-54$

Schramel J, Nagel C, Auer U, Palm F, Aurich C and Moens Y 2012a Distribution of ventilation in pregnant Shetland ponies measured by Electrical Impedance Tomography Respiratory physiology \& neurobiology 180 258-62

Schramel J, van den Hoven R and Moens Y 2012b In vitro validation of a new respiratory ultrasonic plethysmograph Veterinary Anaesthesia and Analgesia 39 366-72

Seaman J, Erickson B K, Kubo K, Hiraga A, Kai M, Yamaya Y and Wagner P D 1995 Exercise induced ventilation/perfusion inequality in the horse Equine veterinary journal 27 104-9

Van Erck-Westergren E, Franklin S H and Bayly W M 2013 Respiratory diseases and their effects on respiratory function and exercise capacity Equine veterinary journal 45 376-87 
Victorino J A, Borges J B, Okamoto V N, Matos G F, Tucci M R, Caramez M P, Tanaka H, Sipmann F S, Santos D C, Barbas C S, Carvalho C R and Amato M B 2004 Imbalances in regional lung ventilation: a validation study on electrical impedance tomography American Journal of Respiratory and Critical Care Medicine

$169791-800$

Young S S and Hall L W 1989 A rapid, non-invasive method for measuring total respiratory impedance in the horse Equine veterinary journal 21 99-105

Zhao Z, Moller K, Steinmann D, Frerichs I and Guttmann J 2009 Evaluation of an electrical impedance tomography-based Global Inhomogeneity Index for pulmonary ventilation distribution Intensive care medicine 35 1900-6 
Table 1 Results of functional image analysis of EIT data recorded from nine standing horses. Two way RM-ANOVA revealed that removal of the inverse respiratory signals (Panel B) decreased the left/right $(\mathrm{L} / \mathrm{R})$ ratio and the global inhomogeneity (GI) indices but increased the centre of ventilation (COV). Please see more explanation in the text.

\begin{tabular}{|c|c|c|c|c|c|c|}
\hline \multirow[b]{2}{*}{ Treatments } & \multicolumn{2}{|c|}{$\mathrm{L} / \mathrm{R}$ ratio } & \multicolumn{2}{|c|}{ COV (\%) } & \multicolumn{2}{|c|}{ GI index } \\
\hline & mean & SD & mean & SD & mean & SD \\
\hline Baseline-1 & 0.80 & 0.11 & 46.3 & 2.4 & 0.36 & 0.02 \\
\hline Baseline-2 & 0.79 & 0.09 & 45.3 & 2.3 & 0.36 & 0.02 \\
\hline Baseline-3 & 0.78 & 0.12 & 45.9 & 2.1 & 0.36 & 0.02 \\
\hline Sigh & $0.66^{\mathrm{a}}$ & 0.09 & 45.1 & 1.9 & 0.35 & 0.01 \\
\hline Rebreathing-1 & 0.75 & 0.10 & $44.4^{\mathrm{a}}$ & 2.4 & 0.36 & 0.03 \\
\hline Baseline-4 & 0.82 & 0.11 & 45.7 & 2.5 & 0.36 & 0.02 \\
\hline Sedation-1 & 0.79 & 0.10 & 44.3 & 2.2 & 0.35 & 0.02 \\
\hline Sedation-2 & 0.82 & 0.11 & 44.4 & 2.7 & 0.36 & 0.02 \\
\hline Rebreathing-2 & 0.79 & 0.09 & 45.3 & 1.3 & 0.36 & 0.02 \\
\hline Baseline-1 & 0.77 & 0.12 & 48.8 & 2.6 & 0.35 & 0.01 \\
\hline Baseline-2 & 0.78 & 0.10 & 47.6 & 2.4 & 0.34 & 0.01 \\
\hline Baseline-3 & 0.76 & 0.11 & 48.1 & 2.1 & 0.35 & 0.02 \\
\hline Sigh & $0.63^{\mathrm{a}, \mathrm{c}}$ & 0.09 & $46.2^{\mathrm{a}, \mathrm{c}}$ & 2.0 & 0.35 & 0.01 \\
\hline Rebreathing-1 & 0.72 & 0.10 & $46.5^{\mathrm{a}}$ & 2.4 & 0.34 & 0.02 \\
\hline Baseline-4 & 0.80 & 0.12 & 48.3 & 2.7 & 0.35 & 0.01 \\
\hline Sedation-1 & 0.79 & 0.11 & $46.0^{\mathrm{a}}$ & 2.5 & 0.34 & 0.02 \\
\hline Sedation-2 & 0.81 & 0.12 & 46.5 & 3.1 & 0.34 & 0.02 \\
\hline Rebreathing-2 & 0.77 & 0.10 & 47.0 & 1.4 & 0.35 & 0.02 \\
\hline
\end{tabular}




\section{Figures}

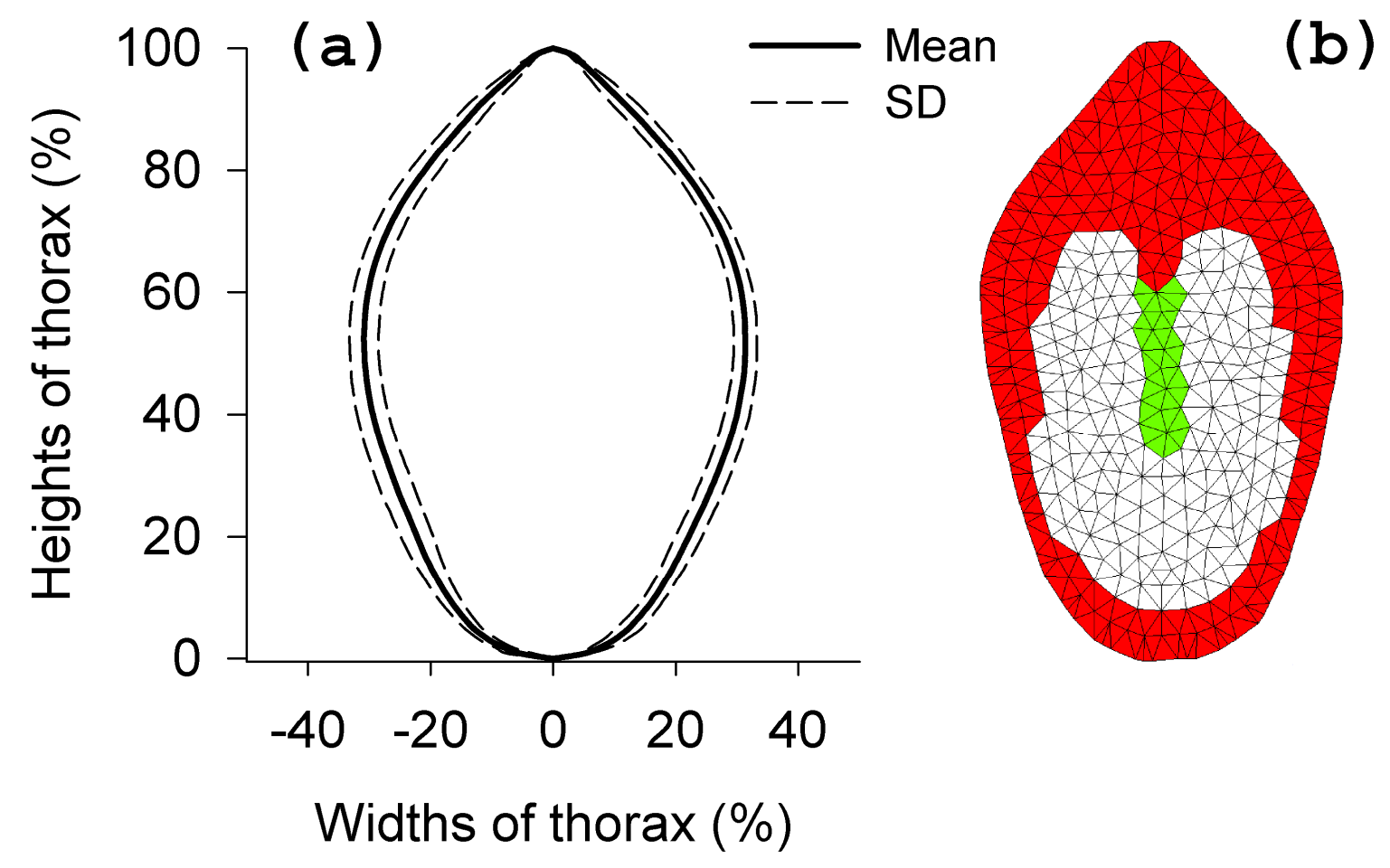

Fig. 1. (a) Mean (SD) of thoracic contours of nine standing adult warm-blood horses. (b) One representative horse contour was used to create an EIT ventilation mesh containing internal organ assumptions. White colour on the mesh represent areas where large impedance variations are expected (corresponding to the location of lungs), moderate impedance changes are expected over the green area (heart and mediastinum) and negligible impedance changes are expected over the red area (muscles, bones etc.). 

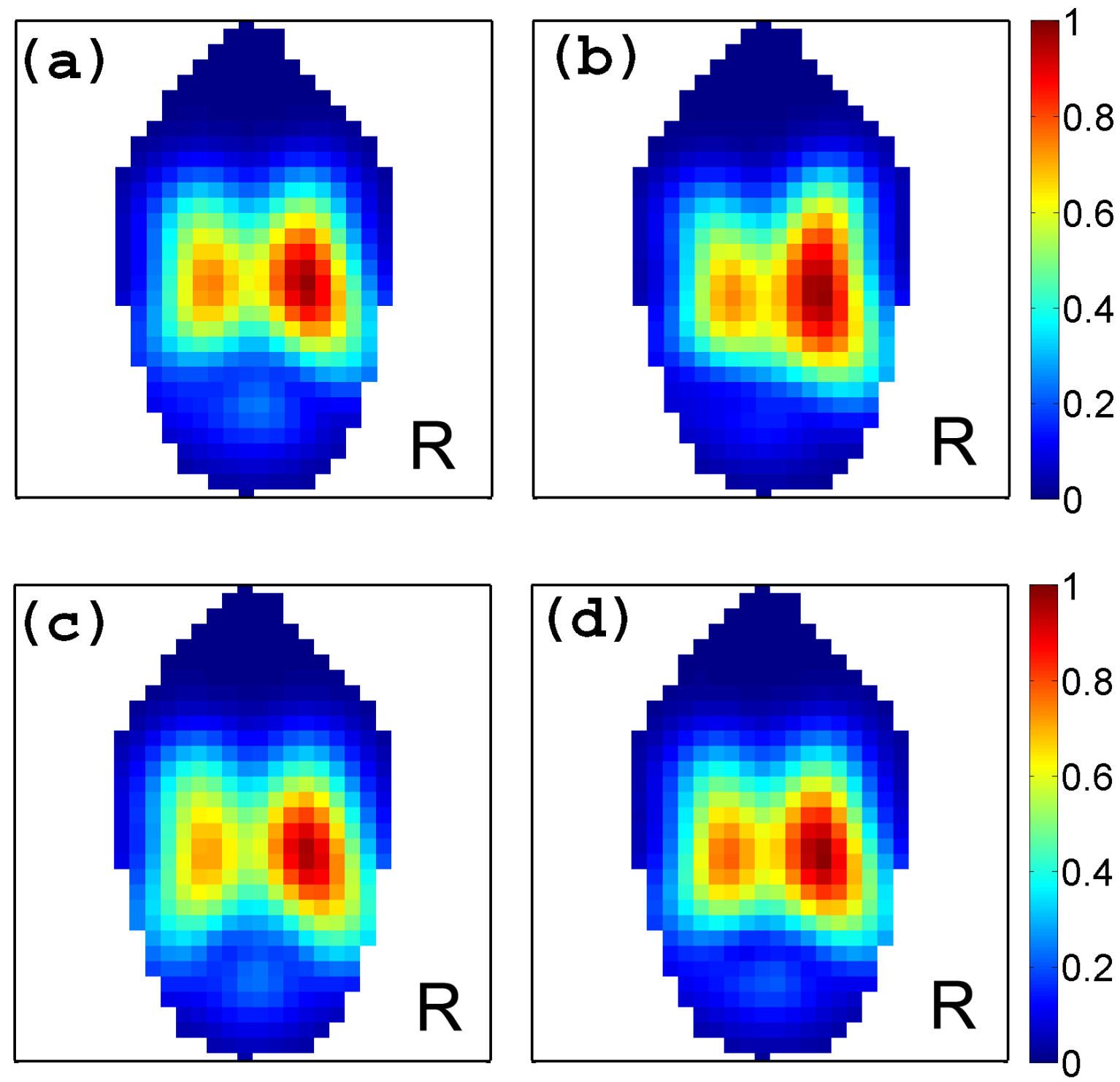

Fig. 2. Functional EIT images depicting pixel-wise SD of impedance changes averaged across nine horses and treatments: (a) baseline, (b) sigh, (c) sedation, (d) rebreathing. Letter ' $R$ ' indicates the right side of the thorax and dorsal direction is upwards. 

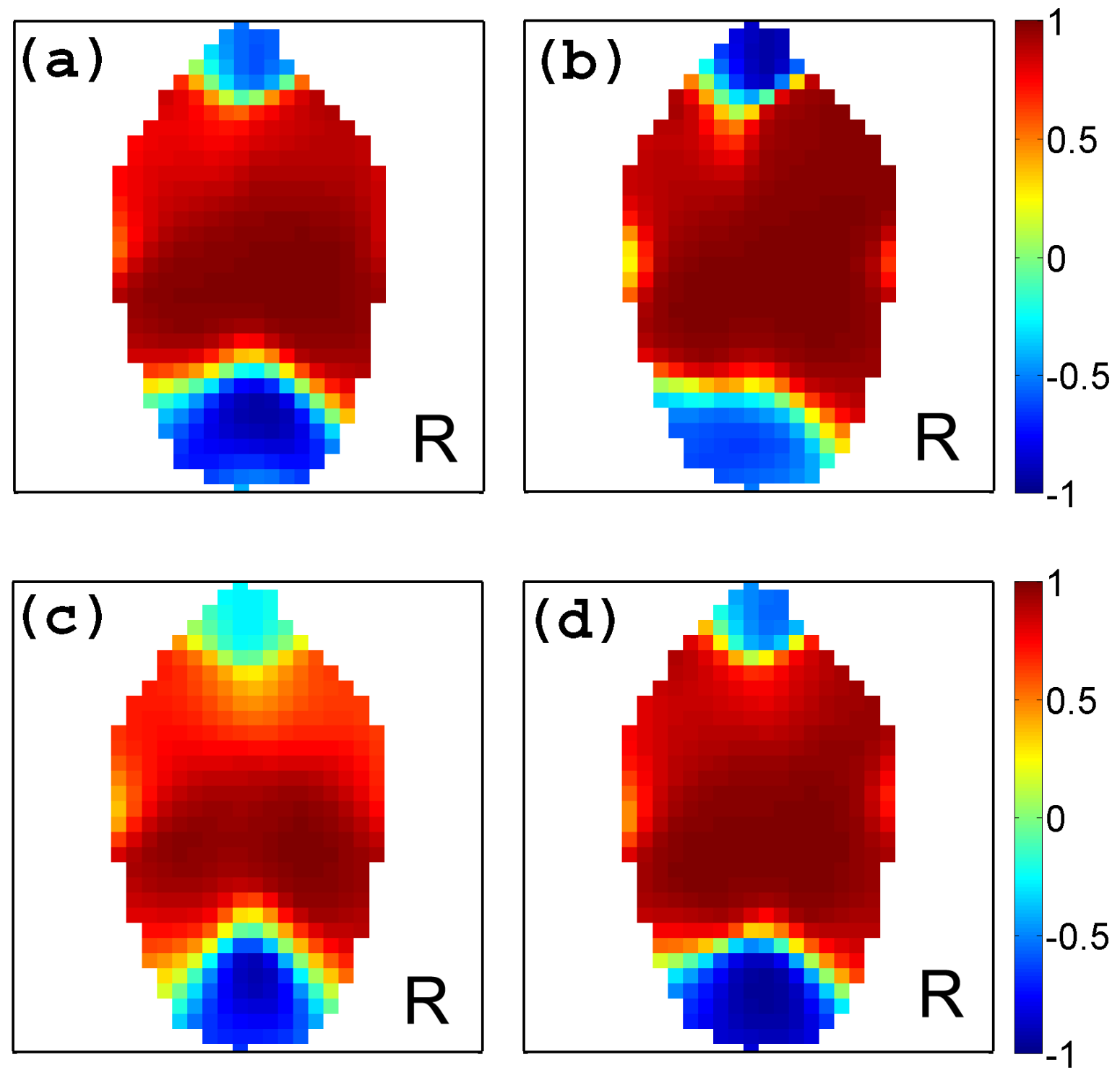

Fig. 3. Functional EIT images depicting the regression coefficient (Pearson R) for each pixel that significantly correlated with the reference respiratory signal. The $\mathrm{R}$ values in each pixel are averaged across nine horses and treatments: (a) baseline, (b) sigh, (c) sedation, (d) rebreathing. Letter ' $\mathrm{R}$ ' indicates the right side of the thorax and dorsal direction is upwards. 


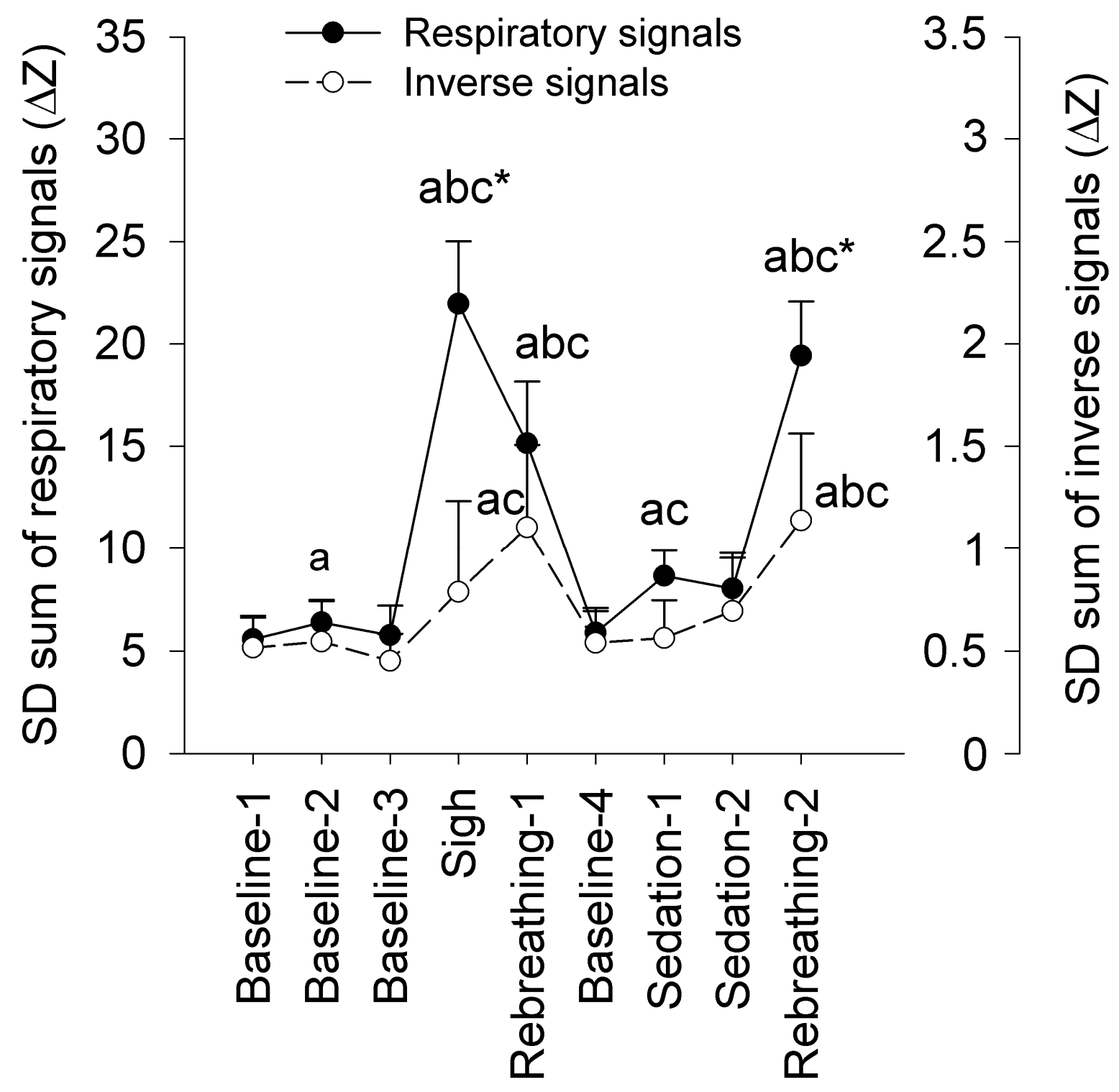

Fig. 4. Mean (SD) of the sums of the pixel SDs of the respiratory and the inverse respiratory signals. a, b or c depicts significant differences form Baseline 1, 2 or 3 respectively. 


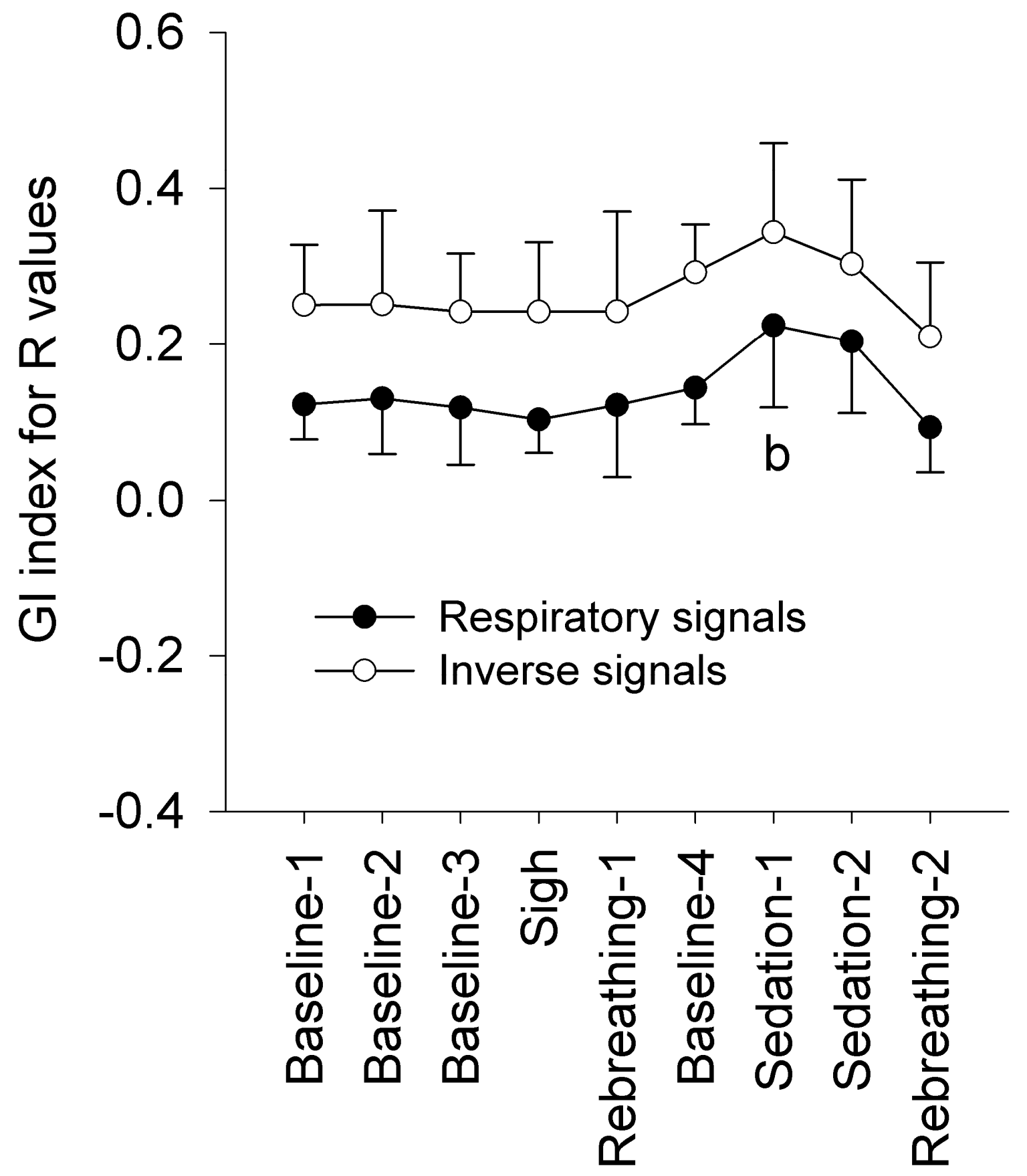

Fig. 5. Mean (SD) of the global inhomogeneity indices of the respiratory and the inverse signals. b depicts significant differences form Baseline-2. 

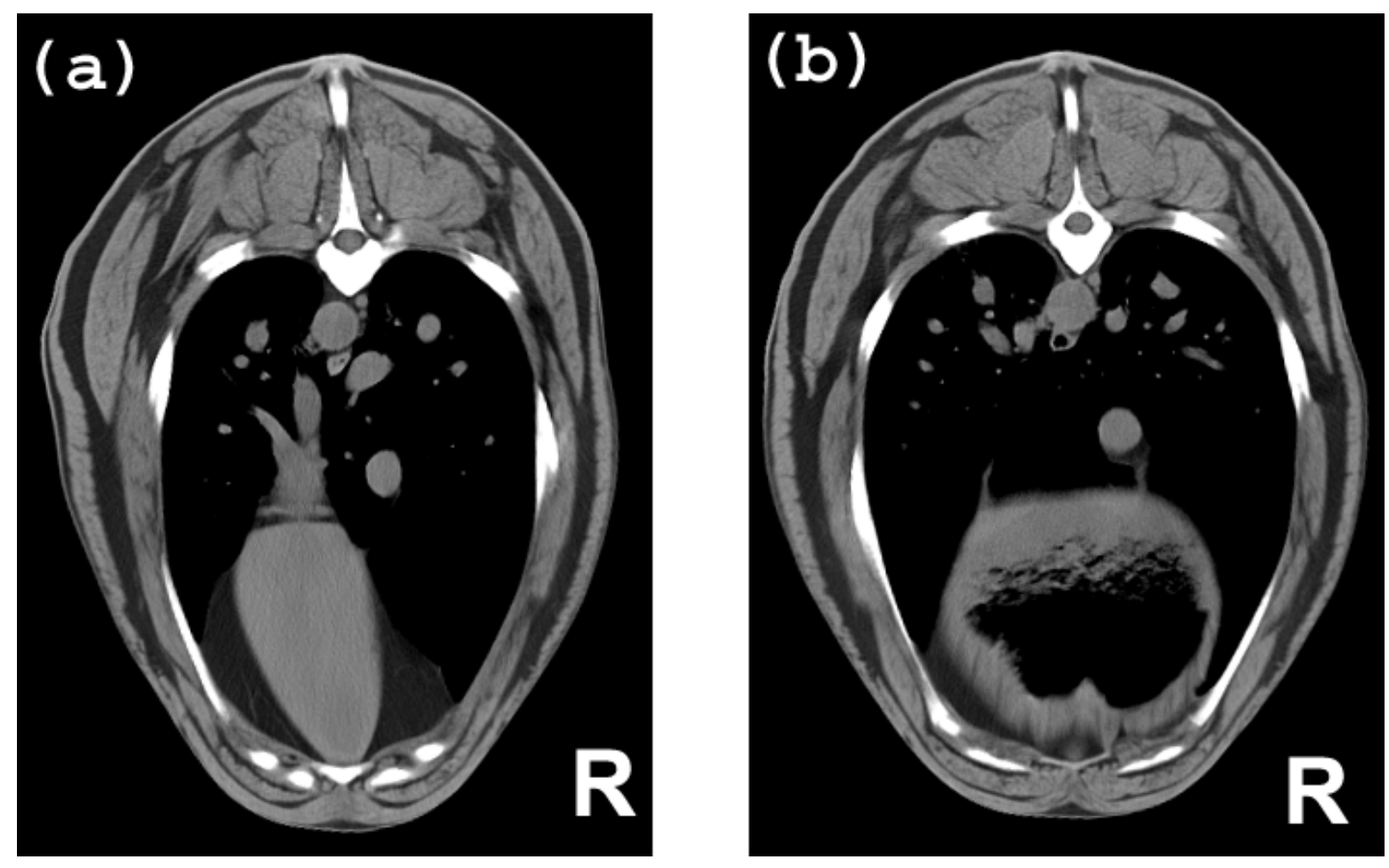

Fig. 6. Thoracic CT images ( $5 \mathrm{~mm}$ thick) of an 8 year old, $100 \mathrm{~kg}$ Shetland pony under intravenous anaesthesia in dorsal recumbency while breathing spontaneously at $0.5 \mathrm{FiO}_{2}$ (Reich et al., 2014). The silhouette of the heart is visible on the ventral part of the image (a) and it is replaced by the silhouette of the gas-filled colon on image (b). These images were recorded $2 \mathrm{~cm}$ apart from each other. Letter ' $R$ ' indicates the right side of the thorax and dorsal direction is upwards. 